\title{
Initial texture effects on the thermal stability and grain growth behavior of nanocrystalline Ni thin films
}

\author{
Josh Kacher ${ }^{*}$, Khalid Hattar ${ }^{2}$, Ian Robertson ${ }^{3}$ \\ ${ }^{1}$ Georgia Institute of Technology, Atlanta, GA \\ ${ }^{2}$ Sandia National Laboratories, Albuquerque, NM \\ ${ }^{3}$ University of Wisconsin - Madison \\ *Corresponding author
}

Tel: (404)894-2781

Email: josh.kacher@mse.gatech.edu

\begin{abstract}
Nanocrystalline Ni thin films with different as-deposited textures were synthesized by varying the substrate temperature during pulsed-laser deposition. The influence of initial texture variations on the thermal stability and annealing behavior of the thin films was studied via in situ transmission electron microscopy annealing and transmission Kikuchi diffraction analysis. It was found that, as the substrate temperature during deposition was increased, the initial microstructure varied from a random distribution of grains to one almost entirely composed of (001) - oriented grains, with the volume fraction of (001) - oriented grains increasing with increasing substrate temperature. The $(001)$ - oriented grains increased thermal stability and constrained grain growth during annealing, resulting in a bimodal grain size distribution in the annealed films. Despite different surface orientations after annealing, the misorientation distribution functions for the films were similar, suggesting that the boundary state is a primary factor in dictating equilibrium.
\end{abstract}

Keywords: Abnormal grain growth; Nanocrystalline; In situ transmission electron microscopy; Transmission Kikuchi diffraction; EBSD

\section{Introduction}

With their promise of superior properties such as improved mechanical strength [1-3] and fatigue resistance [4, 5], nanocrystalline materials continue to be an area of active research. However, due to the high volume-fraction of grain boundaries in comparison to coarse-grained materials, these materials tend to be unstable even at low homologous temperatures, resulting in significant grain coarsening over relatively short time periods [6-15]. Efforts to understand this growth, including strategies to stabilize nanocrystalline structures or promote the development of favorable microstructures, promise to provide greater control in the development and application of nanocrystalline materials [16-18]. For example, experimental work has shown that inducing a bimodal grain size distribution [19] or generating a high density of nanotwins [20, 21] can 
increase the ductility of a sample while still maintaining the strength associated with nanocrystalline materials.

Extensive work using tools such as in situ and post mortem transmission electron microscopy (TEM) characterization [7, 8, 10, 11, 22], transmission Kikuchi diffraction (TKD) analysis [23], and x-ray characterization [6] has shown that grain growth in nanocrystalline materials proceeds in an abnormal manner, with individual grains growing at rates many times higher than grains in the surrounding nanocrystalline matrix. These studies have also demonstrated that grain growth in thin films stagnates at smaller sizes than what is seen in bulk materials. The reasons for the stabilization of the microstructure, that is, the stagnation of grain growth, is still being debated [24], though possible factors include: solute particle pinning [25, 26], surface and texture effects including grain boundary grooving [27-32], relative grain boundary mobilities [33], and vacancy buildup [34]. Previous studies have suggested that grain boundary characteristics, specifically grain boundary complexions, strongly influence the stability and abnormal grain growth behavior of the nanocrystalline materials [35-37].

Ongoing research efforts seek a more fundamental understanding of the factors influencing lowtemperature grain growth that will enable controlled growth behavior and/or increased thermal stability. Many of these efforts have focused on chemical means to stabilize the matrix, specifically through the addition of solute elements or finely dispersed particles that can have a Zener pinning effect on grain boundary growth [16, 38-41]. One area that has received comparatively little attention is the manipulation of initial texture conditions to influence later growth behavior. Computational results have suggested that seeding a grain structure with a strong orientation component can directly influence grain growth kinetics and delay equilibrium [42-46]. Experimentally, little has been done to investigate how variations in the initial texture conditions can influence later growth behavior.

This paper reports experimental results on the influence of initial texture conditions on the thermal stability and annealing behavior of nanocrystalline thin films. It provides experimental confirmation of computational results related to grain boundaries and thermal stability and offers additional insight into the influence of surface energetics on grain growth behavior. The results demonstrate the potential of influencing initial texture in thin films as a tool to increase thermal stability and dictate grain growth behavior in nanocrystalline materials.

\section{Experimental Methods}

Nanocrystalline $\mathrm{Ni}$ samples were made using pulsed-laser deposition at Sandia National Laboratories following a procedure described previously [9, 22, 47]. The samples were deposited on a base of (001) oriented rock salt with a beam energy of $500 \mathrm{~mJ}$ to a final thickness of approximately $70 \mathrm{~nm}$. The growth chamber achieved a base pressure of approximately $5 \times 10^{-7}$ Torr. A $99.97 \%$ pure Ni target was used during the deposition. The Ni was deposited at a nominal laser pulse frequency of $35 \mathrm{~Hz}$ until the desired thickness was achieved. The substrate was maintained at three different temperatures, $-150^{\circ} \mathrm{C}$, room temperature, and $200^{\circ} \mathrm{C}$, during 
deposition. In the remainder of this paper, these samples are referred to as $-150^{\circ} \mathrm{C}$, RT, and $200^{\circ} \mathrm{C}$ films, respectively.

The samples were prepared for TEM and TKD analysis by scoring the surface in a $2 \times 2 \mathrm{~mm}$ grid pattern and submersing the substrates with the films into deionized water. The foils floated to the surface as small squares, from which they were lifted using $3 \mathrm{~mm} \mathrm{Cu}$ grids. In situ TEM annealing and characterization was performed in a Philips CM 300 TEM operated at $300 \mathrm{kV}$. A Gatan-model heating holder was used to anneal the samples. Temperature ramping occurred in stages, $0-200^{\circ} \mathrm{C}, 200-350^{\circ} \mathrm{C}$, and $350-450^{\circ} \mathrm{C}$, and the film was allowed to stabilize between stages. Grain growth was recorded digitally using a CCD camera at 3 frames per second.

Orientation mapping of the pre- and post-annealed films was performed in an FEI XL30 scanning electron microscope using TKD (also known as transmission EBSD or tEBSD). TKD, originally developed by Geiss and Keller [48] and since applied to a number of material systems [49-52], relies on the same principles as EBSD to recover local crystal orientation, but collects forward scattered electrons from thin films rather than backscattered electrons. This variation has been shown to improve the spatial resolution to below $10 \mathrm{~nm}$ [50]. The TKD setup used in this study is the same as that outlined previously [23]. The beam was kept at an accelerating voltage of $30 \mathrm{kV}$ to maximize spatial resolution. All scans were acquired over a $2 \times 2 \mu \mathrm{m}$ area with a $5 \mathrm{~nm}$ step size. All points indexed with low confidence were filtered from the scans but no data interpolation was used except for when calculating the misorientation distribution functions. The thinness of the foils, and possibly relief of internal stress from the mismatch strain between the salt substrate and the Ni film, led to wave formations, altering the surface normal. To correct for this, the sample orientation was adjusted post-acquisition by aligning the expected (001) with the center of the 001 pole figure (this process is illustrated in Figure 1). This correction does not affect characterization of the grain boundaries, only the surface normal orientation. Characterization of the films before and after annealing was done on different sample locations due to the difficulty of relocating previous scan sites. Orientation maps, pole figures, and misorientation distribution functions were generated using EDAX/TSL OIM analysis software.
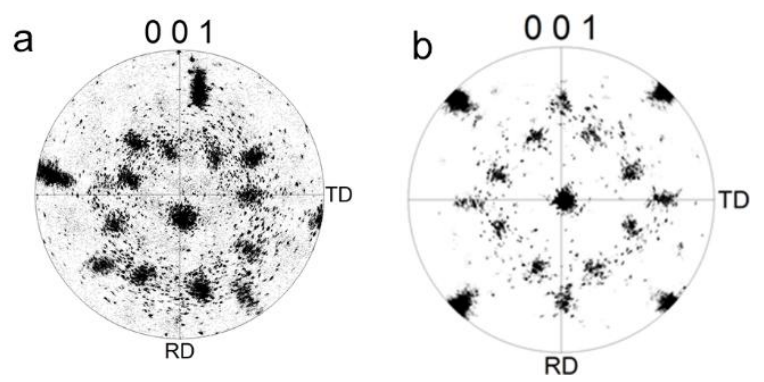

Figure 1. Pole figure of raw data collected from annealed Ni film (a). Pole figure after low confidence points filtered out and (001) plane aligned (b).

\section{Results}




\section{TEM}

Thermal stability of the films, the temperature at which no further grain growth was observed, was reached by $350^{\circ} \mathrm{C}$ for the film deposited at $-150^{\circ} \mathrm{C}$ (i.e. no further microstructural evolution occurred when the temperature was increased to $450^{\circ} \mathrm{C}$ ). Thermal stability was seen at $450^{\circ} \mathrm{C}$ for the film deposited at RT. This stabilization was reached after approximately 2 minutes annealing at temperature. The $200^{\circ} \mathrm{C}$ film remained stable throughout the annealing process. Bright-field micrographs of the films before and after annealing with their associated diffraction patterns are shown in Figures 2, 3, and 5. Videos of the annealing process are also provided (Suppl. Vid. 12).

In Figure 2a-b, it is seen that the initial nanocrystalline structure of the $-150^{\circ} \mathrm{C}$ film is composed of randomly oriented grains with sizes varying from approximately 5-30 $\mathrm{nm}$. The video of the annealing process (Suppl. Vid. 1) shows that abnormal grain growth initiates from dispersed nucleation sites and quickly grows to consume the surrounding nanocrystalline matrix. After annealing, the nanocrystalline matrix was replaced almost entirely by grains in the larger end of the nanograin spectrum and ultrafine grains with grain sizes ranging from 40-500 nm. Almost no grains with diameter below $10 \mathrm{~nm}$ were left in the matrix. Although the majority of the grain boundaries are curved, a few are straight, suggesting a population of coherent $\Sigma 3$ grain boundaries (an example is highlighted in Fig. 2c). This was not confirmed in the TEM analysis, though the orientation mapping results discussed later suggest a similar structure. Very few nanotwins were observed to form in grain interiors. The post-annealing diffraction pattern taken from the same area shows a reduction in the number of grains, the points are more discretized, but no strong texture development is readily apparent.

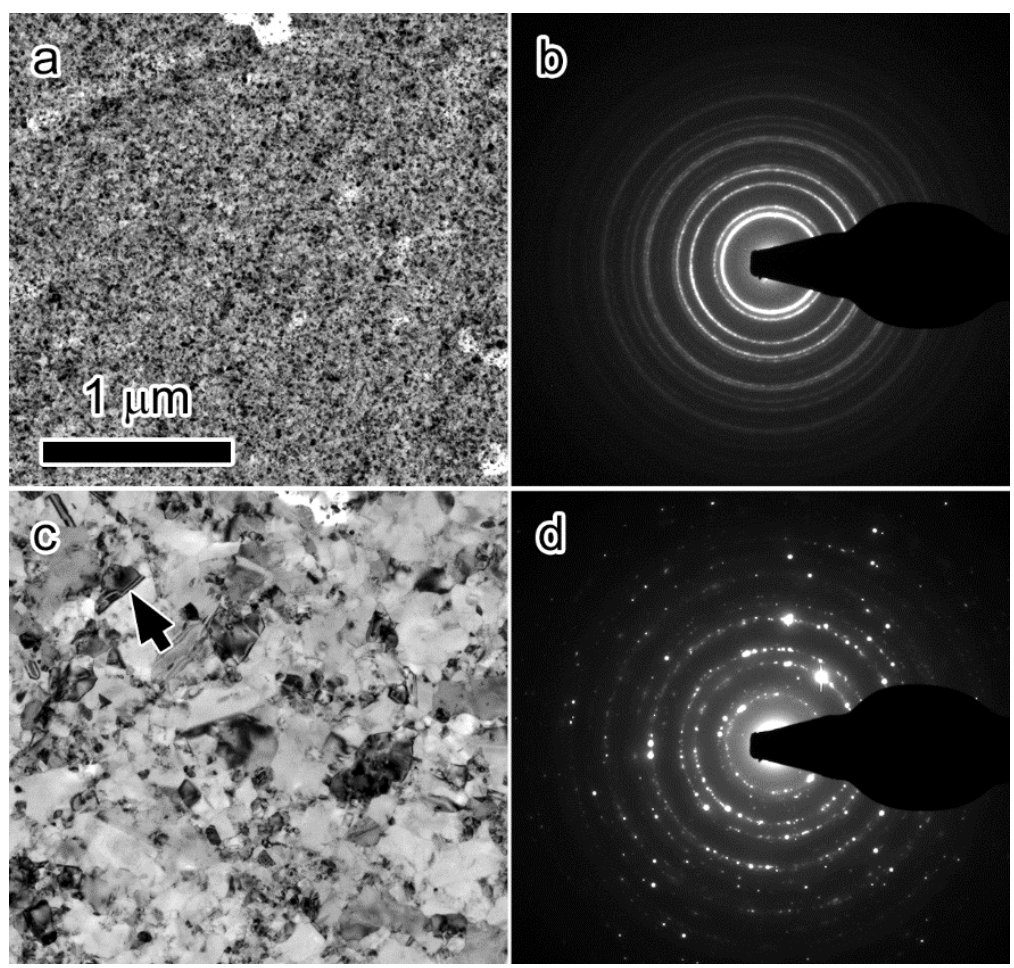


Figure 2. Bright-field TEM micrograph and associated selected area diffraction pattern before (a-b) and after (c-d) annealing of the film deposited at $-150^{\circ} \mathrm{C}$. Arrowhead in (c) indicates a straight boundary discussed in the text.

Figure 3 shows the pre and post-annealing structure of the RT film. Similar to the $-150^{\circ} \mathrm{C}$ film, the initial structure is composed of nanograins in the 5-30 nm size range. The initial microstructure, however, shows strong texture components prior to any annealing (Fig. 3b). During annealing, the film exhibited general grain coarsening as well as abnormal growth of a few grains (Vid 2; example highlighted in Fig. 3c). These abnormal grains grew quickly, reaching sizes of $0.5-2 \mu \mathrm{m}$, about 10 times the average grain size in the surrounding matrix. The aspect ratios of these grains was consistently found to lie between 2-3, suggesting that the growth is constrained in the matrix. The post-annealing diffraction pattern varies little from the preannealing pattern, suggesting that the grain growth did not affect the volume fraction of the different orientations.

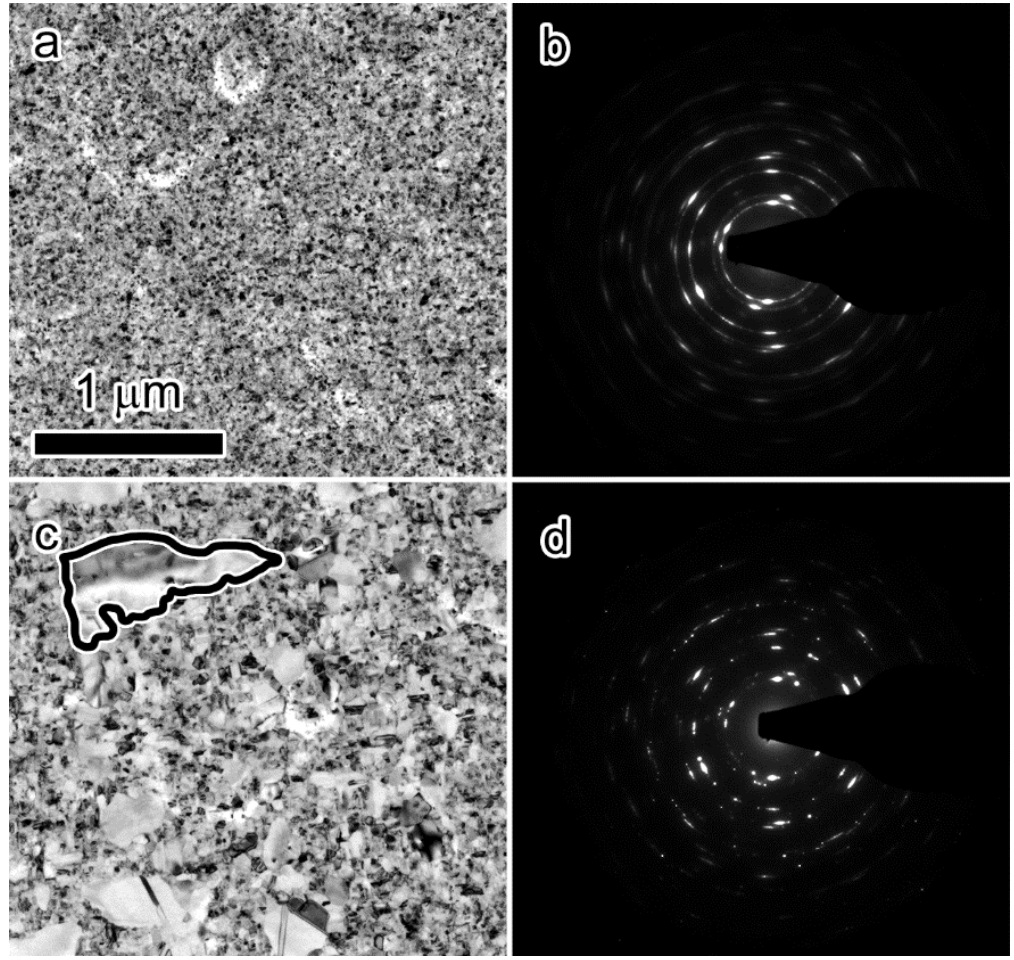

Figure 3. Bright-field TEM micrograph and associated selected area diffraction pattern before (a-b) and after (c-d) annealing of the film deposited at room temperature. An example of an abnormally grown grain is outlined in (c).

Another example of abnormal grain growth in the post-annealing RT film is highlighted in Figure 4. As can be seen, the majority of the grains surrounding the large grain are well below $100 \mathrm{~nm}$ in size, suggesting little grain growth has taken place. Other notable features in the image include an instance of a twin forming during growth, highlighted by arrowhead I, and contrast variations in the interior of the large grain suggestive of stacking fault tetrahedra and loop defects, indicated by arrowhead II. Previous work has suggested that point, line, and volume 
defects form in grain interiors during grain growth to compensate for the low-density boundaries being absorbed [7, 9].

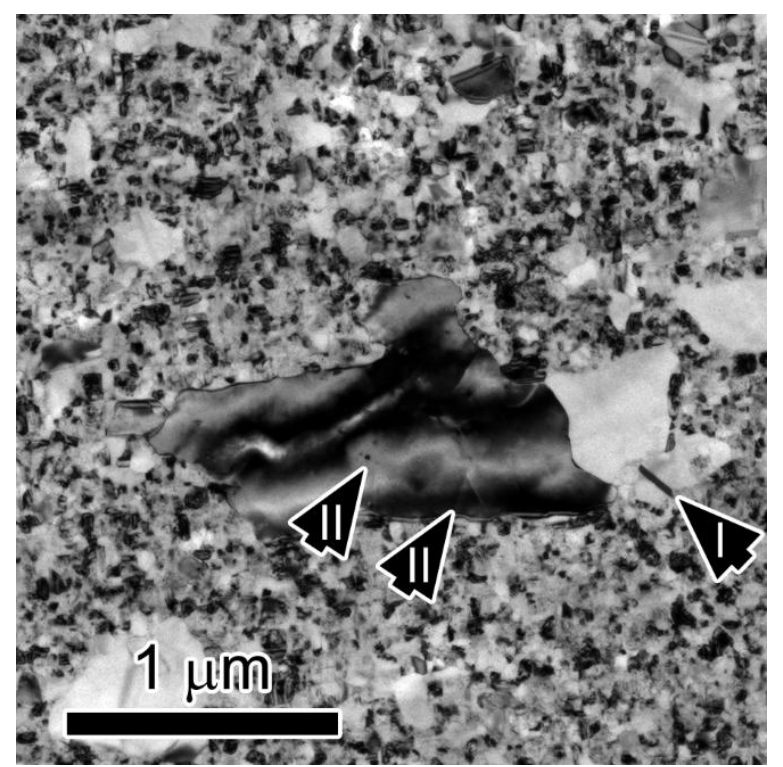

Figure 4. Example of abnormal grain growth post-annealing in the RT deposited film. Arrowhead labeled ' $I$ ' indicates an annealing twin and arrowheads labeled 'II' highlight potential dislocation loop and a stacking fault tetrahedron in the grain interior.

Before annealing, the $200^{\circ} \mathrm{C}$ film did not exhibit a discrete grain boundary structure (Fig. 5a). Contrast variations in the bright-field imaging suggested islands of different character formed, but the associated diffraction pattern suggests that the film is composed of a single orientation. Film bending during the annealing process varied the contrast of the film, but did not result in the observed formation or growth of new grains. The inset in Figure 5d shows the diffraction pattern taken from the region shown in Figure $5 \mathrm{c}$, but tilted on-axis. Indexing the diffraction spots revealed this to be a 001 zone axis. 


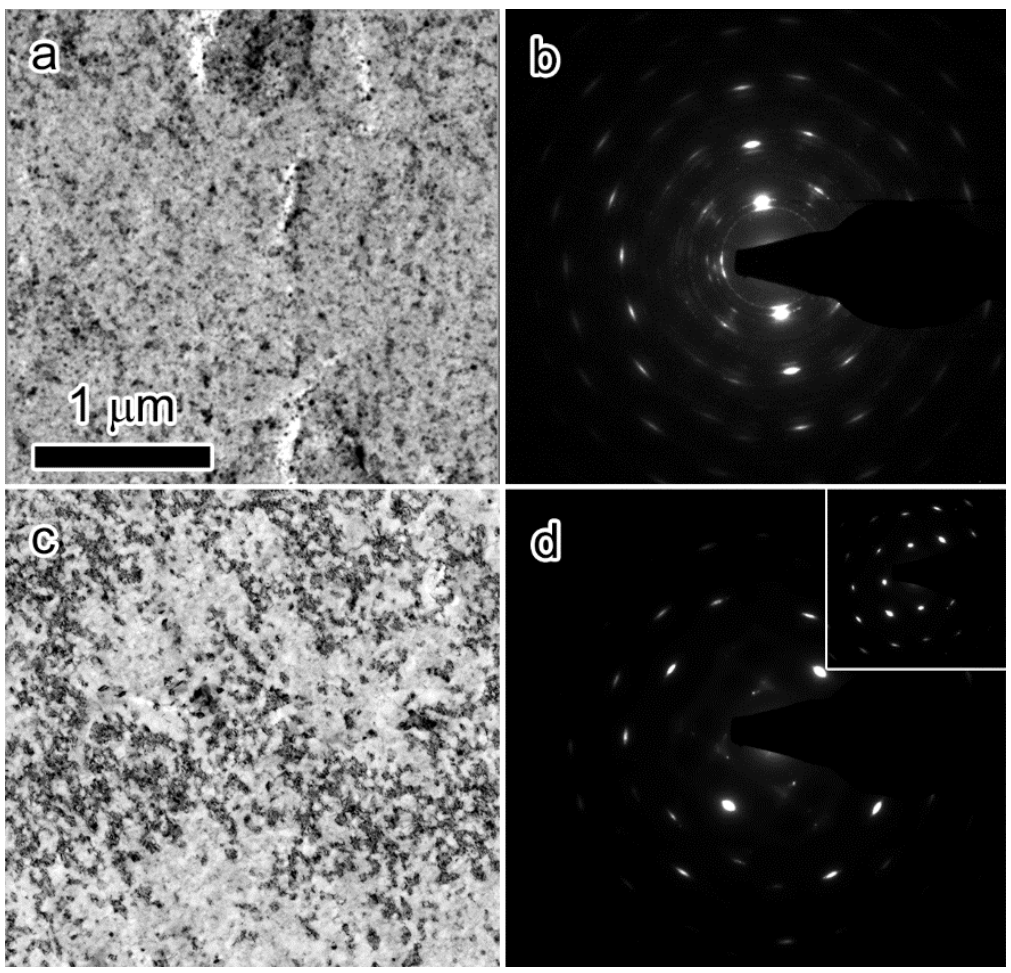

Figure 5. Bright-field TEM micrograph and associated selected area diffraction pattern before (a-b) and after (c-d) annealing of the film deposited at $200^{\circ} \mathrm{C}$. Inset in (d) shows a diffraction pattern taken from the same region but tilted on-axis.

\section{TKD}

TKD scans taken before and after annealing with accompanying pole figures are shown in Figure 6. In the samples deposited at room temperature and $-150^{\circ} \mathrm{C}$, prior to annealing, the majority of the grains were below the resolution limit of TKD, though the limited number of grains resolved suggest a transition from a $\{111\}$ to an (001) texture with increasing temperature (Fig. 6a,d). This becomes more apparent in the sample deposited at $200^{\circ} \mathrm{C}$ where the foil appears near single crystalline with a dominant (001) orientation (Fig. 6g). It should be noted that the pre- and postannealing scans are not from the same region of the film. All pole figures relate to the postannealing structure. 

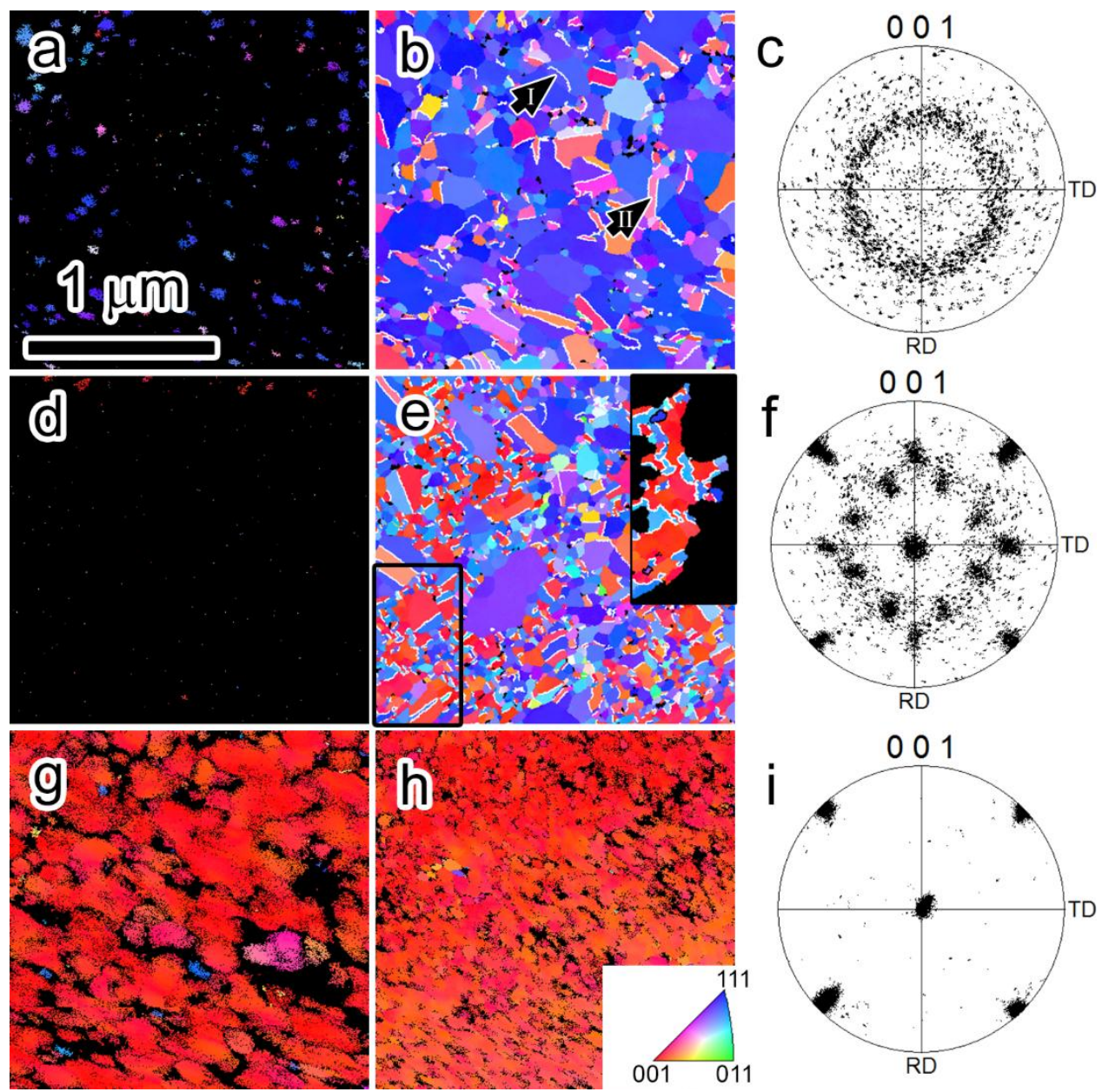

Figure 6. Inverse pole figure (IPF) maps of pre- (column 1) and post- (column 2) annealing thin films with associated pole figures (column 3). a-c) Film deposited at $-150^{\circ} \mathrm{C}$. Labelling in (b) is for reference in the text. d-f) Film deposited at RT. Inset in (e) shows the isolated grain boxed in the same image. g-i) Film deposited at $200^{\circ} \mathrm{C}$. Twin boundaries are highlighted white in the IPF maps. The legend for the IPF maps is included in (h).

The initial microstructure of the $-150^{\circ} \mathrm{C}$ film was composed primarily of nanograins with sizes below the resolution of TKD analysis (Fig. 6a). A few exceptions in the form of $\{111\}$ - oriented grains existed with grain sizes on the order of $10 \mathrm{~nm}$. Post-annealing, the $-150^{\circ} \mathrm{C}$ films developed a strong $\{111\}$ - fiber texture. This texture is evident from the outer ring in the pole figure shown in Figure 6c. These grains were populated by a high twin density composed of two sets of twins. The first, those related to their parent grains by a rotation about the surface plane normal, were also $\{111\}$ - oriented. An example of this is labeled 'I' in Figure 6b. The second category of twins were related to their parent grains by a rotation axis inclined to the surface normal and developed a near - $\{001\}$ - orientation. An example of this is labeled 'II' in Figure 6b. This second category of twin resulted in the inner ring, near the center, in the associated pole figure (Fig. 6c).

In the RT film, the initial microstructure was similarly composed of nanograins with only a few grains resolvable by TKD (Fig. 6d). These grains, however, were primarily (001) - oriented, 
rather than the $\{111\}$ orientation existing in the pre-annealing $-150^{\circ} \mathrm{C}$ films. Post annealing, orientations similar to those seen in $-150^{\circ} \mathrm{C}$ dominate the film but their distribution is much different (Fig. 6e-f). Here, the texture is divided three ways between the (001) orientation, the primary orientation seen in the initial microstructure, $\{122\}$ - oriented grains, or the twin variants of the $(001)$ - orientation, and scattered $\{111\}$ - oriented grains. The $(001)$ - orientation is composed of a single orientation and so the twins from the (001) parent grain appear as four distinct variants (one for each of the $\{111\}$ planes in an FCC lattice). Many of these (001) oriented grains contained multiple twin variants. An example of this is shown in the inset in Figure 6e in which a single grain containing three twin variants is isolated. Interestingly, many of these twins are fully enclosed in a single grain.

The $200^{\circ} \mathrm{C}$ film experienced the least texture variation during annealing. As can be seen in Figure $6 \mathrm{~g}$, the initial texture was composed primarily of a single (001) orientation with a few scattered $\{111\}$ - oriented grains. This changed little after annealing with the exception of perhaps greater homogeneity in the film.

Using surface energy calculations by Wen et al. [53], the energy of each grain was correlated with the grain size and the average surface energy of each film was calculated (shown in Fig. 7a). The grain size vs. grain surface energy was plotted for the $-150^{\circ} \mathrm{C}$ and RT films and is shown in Figure 7b-c. From the data, it is apparent that the average surface energy of the annealed samples increases significantly with increasing substrate temperature during deposition. This is largely related to the increasing fraction of (001) - oriented grains at the expense of the lower energy $\{111\}$ - oriented grains (the energies of the two orientations are $1.304 \mathrm{x} 10^{-8} \mathrm{~J} / \mathrm{m}^{2}$ and $1.171 \times 10^{-8} \mathrm{~J} / \mathrm{m}^{2}$, respectively). The plots of grain size vs. surface energy show that, for the $150^{\circ} \mathrm{C}$ film only, there is an inverse correlation between the surface energy and the grain size. That is, as the orientation-dependent surface energy increases, the maximum size grains reach with that orientation decreases, with the largest grains having $\{111\}$ - orientations. Interestingly, the RT film shows no such correlation, with the largest grain having a near-(435) orientation, corresponding to a surface energy of $1.335 \times 10^{-8} \mathrm{~J} / \mathrm{m}^{2}$.
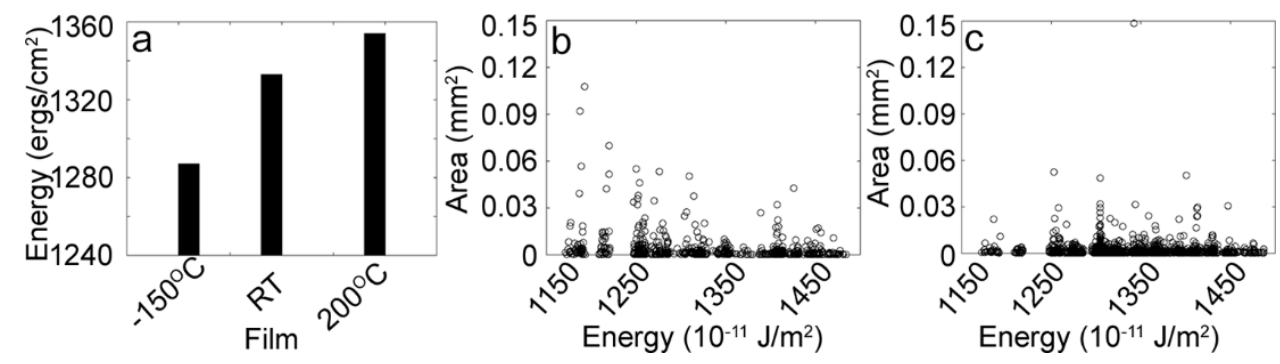

Figure 7. Surface energies of the annealed films. a) Average surface energy of each film. b) Energy vs area of the grains in the $-150^{\circ} \mathrm{C}$ film after annealing (orientation map shown in Fig. 6b). c) Energy vs area of the grains in the RT film after annealing (orientation map shown in Fig. 6e). 
Misorientation distribution functions were generated using a harmonic series expansion (Fig. 8). As can be seen, despite the different textures present in the annealed films, they show qualitatively similar grain boundary characteristics. In both films, there is a disproportionately large fraction of random low-angle grain boundaries and high-angle rotations about the $\{111\}$ axes corresponding to $\Sigma 3$ twin boundaries. The primary differences between the annealed textures occur in the $15-20^{\circ}$ misorientation range, with the RT film showing a lower prevalence of $20^{\circ} / /\{101\}$ boundaries. To accurately calculate grain boundary energies, all five degrees of freedom of the grain boundaries is needed. This information is not available in these twodimensional scans, but calculations of grain boundary energies by Olmsted et al. suggest that the lowest energy boundaries are in general low-angle boundaries and coherent $\Sigma 3$ [54], both of which are abundant in the annealed films.
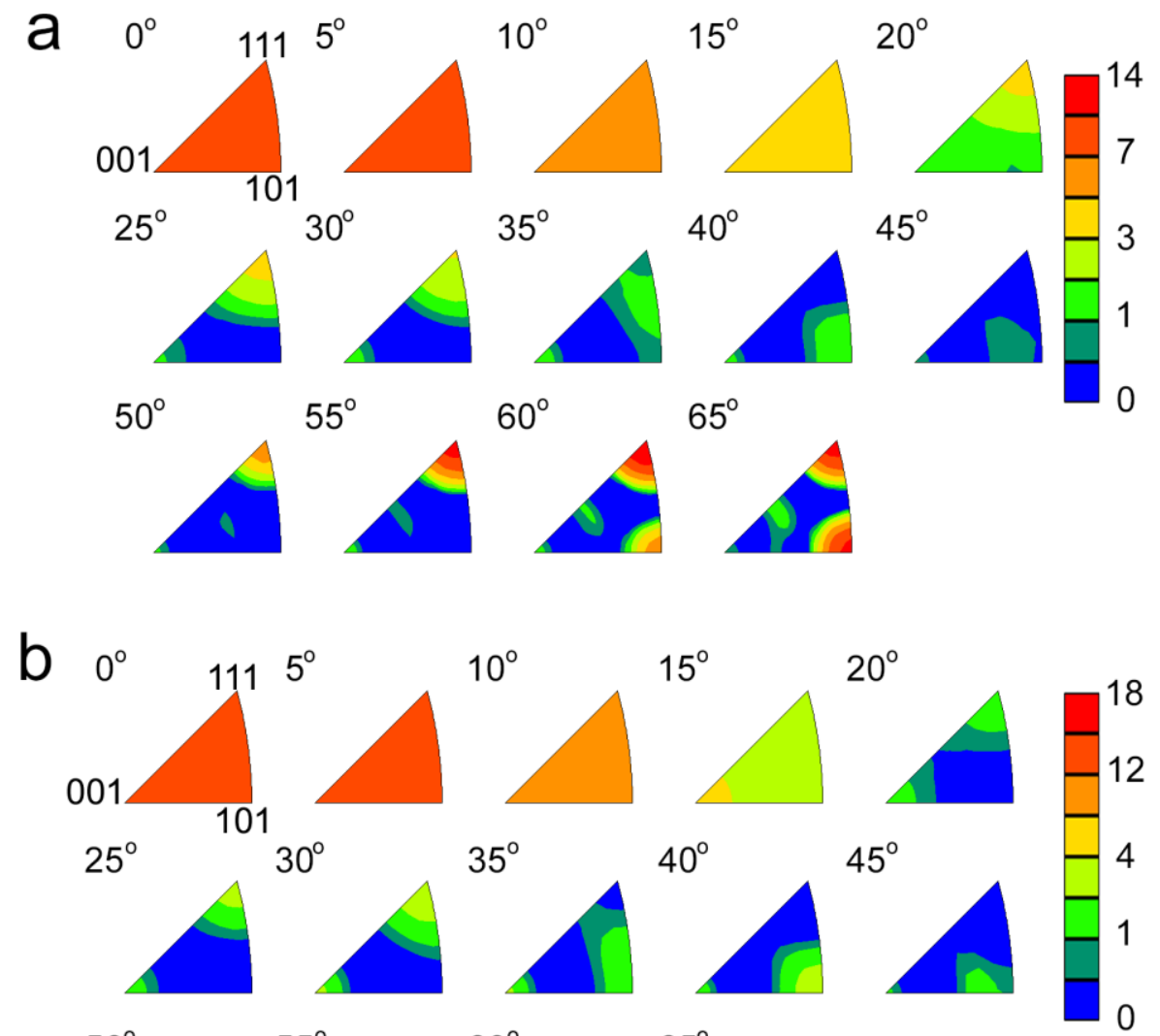

$30^{\circ}$

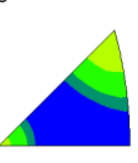

$35^{\circ}$
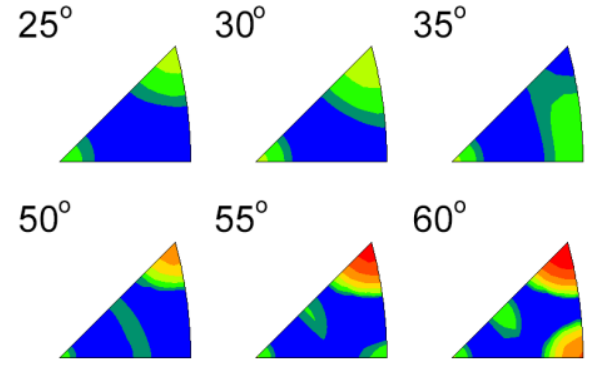

$55^{\circ}$

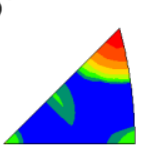

$60^{\circ}$

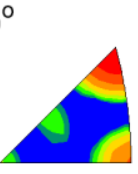

$65^{\circ}$

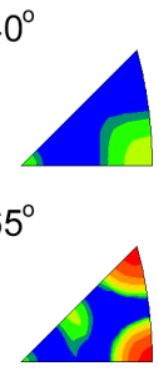

Figure 8. Misorientation distribution function for the annealed $-150^{\circ} \mathrm{C}$ (a) and $\mathrm{RT}$ (b) films. The units of the associated legends are times random.

\section{Discussion}

The results shown in this paper demonstrate the influence of both the surface and grain boundary energies and the effect of initial conditions on the thermal stability and annealing behavior of 
nanocrystalline thin films. Here, we restrict the discussion to the $-150^{\circ} \mathrm{C}$ and RT films as they represent unconstrained and constrained growth conditions, respectively. The as-deposited $150^{\circ} \mathrm{C}$ film had a nominally random initial texture and can be thought of as a homogeneous film with dispersed grain growth initiation sites (shown schematically in Figure 9a). During annealing, abnormal grain growth dominated the microstructural transformation and was driven by both the reduction of surface energy (as evidenced by the prevalence of $\{111\}$ - oriented grains and the inverse grain size to surface energy relationship shown in Fig. 7) and the reduction of boundary energy. Equilibrium was reached in the annealed microstructure only after the majority of the nanocrystalline grains were consumed by the abnormal grains. The RT film, conversely, had a strong initial (001) texture in the as-deposited films. This initial (001) texture increased the thermal stability of the film, raising the temperature at which significant grain growth initiated $100^{\circ} \mathrm{C}$ higher than what was seen in the $-150^{\circ} \mathrm{C}$ film. Throughout the annealing process, the (001) texture remained prominent and restricted the uninhibited growth of lowsurface-energy grains. This process is represented schematically in Figure 9b. The resultant tortuous grain growth path is evidenced by the final grain shape shown in the Figure 6e inset.

a) Unconstrained growth

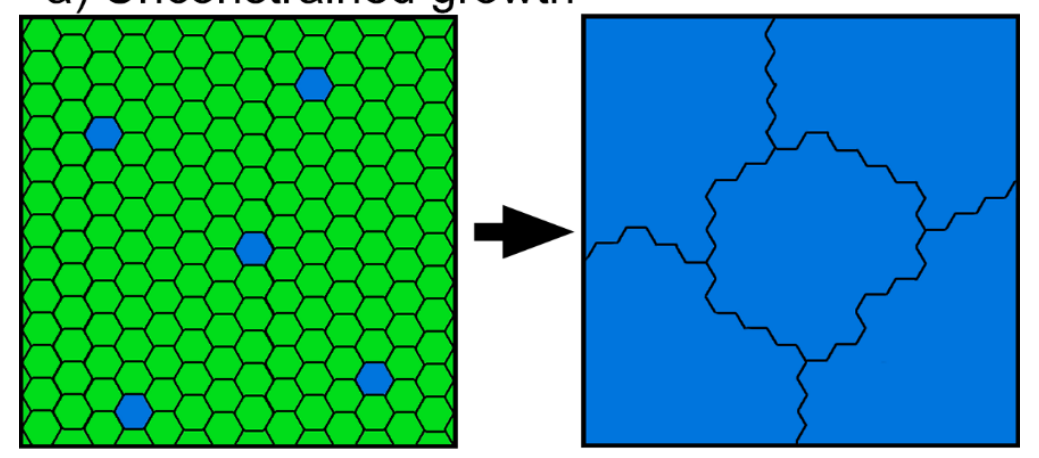

b) Constrained growth

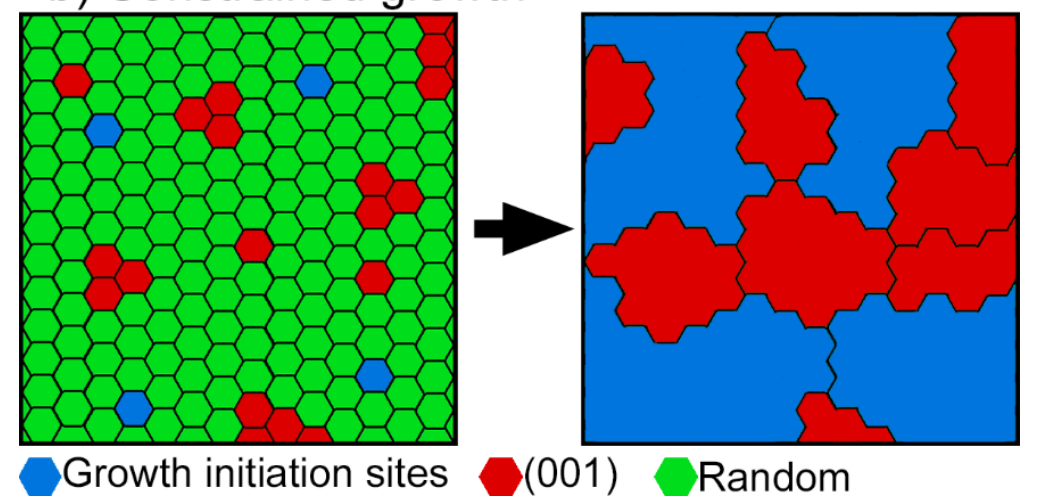

Figure 9. Grain growth schematic showing a) unconstrained growth as the $-150^{\circ} \mathrm{C}$ film experiences and b) constrained growth as is seen in the RT film. The transition from left to right represents the pre and post annealing films. Note that twins are not depicted in this schematic. 
Although the texture evolution during annealing appeared to be driven by both surface and grain boundary energy considerations, the strong similarities in the grain boundary distribution functions of the two films, including the prevalence of low angle and $\Sigma 3$ boundaries, suggest that thermal equilibrium is largely a function of the grain boundary energy.

Several computational studies have investigated the effects of texture on grain growth and stability. Kazaryan investigated normal grain growth in two-dimensional systems [44]. Similar to what was seen in the RT films, they found that grain shape anisotropy increased in textured films during grain growth. However, in contrast to the results presented here, they did not observe a stagnation in grain growth during annealing. Gruber et al. investigated the growth of grains in two and three dimensional system, specifically investigating the evolution of the misorientation distribution function with time [45]. They found that the misorientation distribution function consistently showed an inverse relationship between boundary energy and average area. When an initial texture component was added to the simulation, the misorientation distribution never reached a steady state distribution. This is in contrast to what is reported experimentally in this study as both the $-150^{\circ} \mathrm{C}$ and RT films reached a similar annealed configuration. Ma et al. considered two cases in two dimensional grain growth simulations, random initial texture and a texture with a cube component embedded in a randomly textured matrix [42]. In both cases, they found that the local grain boundary energy density was a key factor in dictating grain growth, with the initial texture influencing grain growth kinetics and clustering behavior but not the driving force. Grain boundary energy density drove the microstructure to favor low energy grain boundaries. These computational studies capture the experimentally observed drive towards low grain boundary energy considerations but do not reflect the equilibrium state, increased thermal stability, and grain growth stagnation of textured films. This disagreement between experiment and model is likely due to surface energy and grain boundary grooving effects, which are present in the experiment but not included in the simulations.

Many experimental and computational studies have reported the thickness-dependent development of $\{111\}$ textures due to surface effects [27, 29, 55, 56]. For example, Floro and Thompson found that surface energy effects can have a large influence on grain growth behavior in thin films, driving the annealed texture towards low surface-energy orientations [57]. Jamshidian et al. modelled isolated (001) and (111) - oriented grains in films of varying thickness [58]. They found that as the thickness increased, boundary migration led to the expansion of the (001) - oriented grain at the expense of the (111) grain. This behavior reversed as the thickness decreased below $\sim 1.2 \mu \mathrm{m}$. As the films investigated in this study were approximately $70 \mathrm{~nm}$ thick, there should be a strong driving force for the $\{111\}$ - oriented grains to grow and replace the (001) - oriented grains. Instead, grain boundaries between the two orientations appeared to be stable, though this may be reflective of the lower temperatures at which the experiments were conducted.

Reasons for twin generation during annealing have been attributed to growth accidents during grain boundary migration [59] and reduction of grain boundary energy [60]. This latter mechanism involves twin generation at triple lines. As the energy cost associated with a coherent twin boundary is low, the overall system energy can be reduced if the new, twinned orientation 
has a lower interface energy with two grains at a triple junction than the original orientation. Inspection of the orientation maps shows that some twins do indeed originate at triple junctions, though many nucleate at grain boundaries or are found in grain interiors, suggesting that growth accidents are a likely explanation in these samples. It is interesting to note here that twins fully enclosed in grain interiors were restricted to (001)-oriented grains, suggesting that these grains are much more prone to growth accidents than $\{111\}$-oriented grains.

\section{Conclusion}

During annealing of nanocrystalline thin films, evolution of the microstructure is dictated by the reduction of both the surface and grain boundary energy. Both influences are readily apparent during grain growth through the reduction of grain boundary area and rapid growth of low surface-energy grains. The equilibrium state of the annealed films, however, appears to be predominately a function of the grain boundary distribution. Prepopulating nanocrystalline thin films with (001) grains was shown to increase the thermal stability of the nanocrystalline structure and restrict growth of low surface-energy grains, increase the prevalence of twin growth accidents, and result in a less equiaxed final grain structure.

\section{Acknowledgment}

The authors would like to thanks R. Goeke and B.G. Clark for useful discussion. JK and IMR acknowledge support by the US Department of Energy Office of Basic Energy Sciences, Division of Materials Science, under award No. DEFG- 02-07ER46443. TKD characterization was done at EDAX-TSL facilities in Draper, Utah. Sandia National Laboratories is a multiprogram laboratory operated by Sandia Corporation, a wholly owned subsidiary of Lockheed Martin company, for the U.S. Department of Energy's National Nuclear Security Administration under contract DE-AC04-94AL85000. KH acknowledges support from the Division of Materials Science and Engineering, Office of Basic Energy Sciences, U.S. Department of Energy at Sandia.

\section{References}

1. Sanders, P.G., J.A. Eastmant, and J. Weertman, Elastic and tensile behavior of nanocrystalline copper and palladium, Acta Materialia, 45 (1997) 4019-4025.

2. Pande, C.S. and K.B. Cooper, Nanomechanics of Hall-Petch relationship in nanocrystalline materials, Progress in Materials Science, 54 (2009) 689-706.

3. Lu, K., L. Lu, and S. Suresh, Strengthening materials by engineering coherent internal boundaries at the nanoscale, Science, 324 (2009) 349-342.

4. Hanlon, T., Y.-N. Kwon, and S. Suresh, Grain size effects on the fatigue response of nanocrystalline materials, Scripta Materialia, 49 (2003) 675-680.

5. Meyers, M.A., A. Mishra, and D.J. Benson, Mechanical properties of nanocrystalline materials, Progress in Materials Science, 51 (2006) 427-556.

6. Ames, M., J. Markmann, R. Karos, A. Michels, A. Tschope, and R. Birringer, Unraveling the nature of room temperature grain growth in nanocrystalline materials, Acta Materialia, 56 (2008) 4255-4266. 
7. Follstaedt, D.M., K. Hattar, J.A. Knapp, and I.M. Robertson, In situ TEM investigation of abnormal grain growth in nanocrystalline nickel, Mater. Res. Soc. Symp. Proc., 907E (2006)

8. Brewer, L.N., D.M. Follstaedt, K. Hattar, J.A. Knapp, M.A. Rodriguez, and I.M. Robertson, Competitive abnormal grain growth between allotropic phases in nanocrystalline nickel, Advanced Materials, 22 (2010) 1161-1164.

9. Hattar, K., D.M. Follstaedt, J.A. Knapp, and I.M. Robertson, Defect structures created during abnormal grain growth in pulsed-laser deposited nickel, Acta Materialia, 56 (2008) 794-801.

10. Hibbard, G.D., K.T. Aust, and U. Erb, On interfacial velocities during abnormal grain growth at ultra-high driving forces, Journal of Materials Science, 43 (2008) 6441-6452.

11. Hibbard, G.D., V. Radmilovic, K.T. Aust, and U. Erb, Grain boundary migration during abnormal grain growth in nanocrystalline Ni, Materials Science \& Engineering A, 494 (2008) 232-238.

12. Kim, B.-K., J.A. Szpunar, and R. Varano, Texture evolution in grain growth of nanocrystalline Ni, Materials Science Forum, 408-412 (2002) 937-942.

13. Kim, S.G. and Y.B. Park, Grain boundary, solute drag and abnormal grain growth, Acta Materialia, 56 (2008) 3739-3753.

14. Da Silva, M., C. Wille, U. Klement, P. Choi, and T. Al-Kassab, Electrodeposited nanocrystalline Co-P alloys: Microstructural characterization and thermal stability, Materials Science and Engineering A, 445-446 (2007) 31-39.

15. Klement, U., U. Erb, A.M. El-Sherik, and K.T. Aust, Thermal stability of nanocrystalline Ni, Materials Science \& Engineering A, A203 (1995) 177-186.

16. Chookajorn, T., H.A. Murdoch, and C.A. Schuh, Design of stable nanocrystalline alloys, Science, 337 (2012) 951-955.

17. Greiser, J., P. Mullner, and E. Arzt, Abnormal growth of "giant" grains in silver thin films, Acta Materialia, 49 (2001) 1041-1050.

18. Gottstein, G., A.D. Rollett, and L.S. Shvindlerman, On the validity of the von NeumannMullins relation, Scripta Materialia, 51 (2004) 611-616.

19. Wang, Y., M. Chen, F. Zhou, and E. Ma, High tensile ductility in a nanostructured metal, Nature, 419 (2002) 912-914.

20. Lu, L., Y. Shen, X. Chen, L. Qian, and K. Lu, Ultrahigh strength and high electrical conductivity in copper, Science, 304 (2004) 422-426.

21. Schute, C.J., B.D. Myers, S. Xie, S.-Y. Li, T.W. Barbee Jr, A.M. Hodge, and J. Weertman, Detwinning, damage, and crack initiation during cyclic loading of $\mathrm{Cu}$ samples containing aligned nanotwins, Acta Materialia, 59 (2011) 4569-4577.

22. Kacher, J., I.M. Robertson, M. Nowell, J. Knapp, and K. Hattar, Study of rapid grain boundary migration in a nanocrystalline Ni thin film, Materials Science and Engineering A, 528 (2011) 1628-1635.

23. Kacher, J., P. Elizaga, S.D. House, K. Hattar, M. Nowell, and I.M. Robertson, Thermal stability of Ni/NiO multilayers, Materials Science \& Engineering A, 568 (2013) 49-60.

24. Barmak, K., E. Eggeling, D. Kinderlehrer, R. Sharp, S. Ta'asan, A.D. Rollett, and K.R. Coffey, Grain growth and the puzzle of its stagnation in thin films: The curious tale of a tail and an ear, Progress in Materials Science, 58 (2013) 987-1055.

25. Le Gall, R., G. Liao, and G. Saindrenan, In-situ SEM studies of grain boundary migration during recrystallization of cold-rolled nickel, Scripta Materialia, 41 (1999) 427-432. 
26. Cahn, J., Coherent fluctuations and nucleation in isotropic solids, Acta Metallurgica, 10 (1962) 907-913.

27. Frost, H.J., C.V. Thompson, and D.T. Walton, Simulation of thin film grain structures. II. Abnormal grain growth, Acta Metallurgica et Materialia, 40 (1992) 779-793.

28. Thompson, C.V., Grain growth in polycrystalline thin films of semiconductors, Interface Science, 6 (1998) 85-93.

29. Thompson, C.V. and R. Carel, Texture development in polycrystalline thin films, Materials Science and Engineering B, 32 (1995) 211-219.

30. Frost, H.J., C.V. Thompson, and D.T. Walton, Simulation of thin film grain structuresI. Grain growth stagnation, Acta Metallurgica et Materialia, 38 (1990) 1455-1462.

31. Mullins, W.W., The effect of thermal grooving on grain boundary motion, Acta Metallurgica, 6 (1958) 414-427.

32. Lou, C. and M.A. Player, Advection-diffusion model for the stagnation of normal grain growth in thin films, Journal of Physics D: Applied Physics, 35 (2002) 1805.

33. Gottstein, G., L.S. Shvindlerman, and B. Zhao, Thermodynamics and kinetics of grain boundary triple junctions in metals: Recent developments, Scripta Materialia, 62 (2010) 914-917.

34. Estrin, Y., G. Gottstein, and L.S. Shvindlerman, Intermittent `self-locking' of grain growth in fine-grained materials, Scripta Materialia, 41 (1999) 385-390.

35. Rupert, T.J., The role of complexions in metallic nano-grain stability and deformation, Current Opinion in Solid State and Materials Science,

36. Dillon, S.J., M.P. Harmer, and G.S. Rohrer, The relative energies of normally and abnormally growing grain boundaries in alumina displaying different complexions, Journal of the American Ceramic Society, 93 (2010) 1796-1802.

37. Raabe, D., M. Herbig, S. Sandlöbes, Y. Li, D. Tytko, M. Kuzmina, D. Ponge, and P.P. Choi, Grain boundary segregation engineering in metallic alloys: A pathway to the design of interfaces, Current Opinion in Solid State and Materials Science, 18 (2014) 253-261.

38. Clark, B.G., K. Hattar, M.T. Marshall, T. Chookajorn, B.L. Boyce, and C.A. Schuh, Thermal Stability Comparison of Nanocrystalline Fe-Based Binary Alloy Pairs, JOM, 68 (2016) 1625-1633.

39. Saber, M., H. Kotan, C.C. Koch, and R.O. Scattergood, Thermodynamic stabilization of nanocrystalline binary alloys, Journal of Applied Physics, 113 (2013) 063515.

40. Darling, K.A., B.K. VanLeeuwen, C.C. Koch, and R.O. Scattergood, Thermal stability of nanocrystalline Fe-Zr alloys, Materials Science and Engineering: A, 527 (2010) 35723580 .

41. Moelans, N., B. Blanpain, and P. Wollants, Pinning effect of second-phase particles on grain growth in polycrystalline films studied by 3-D phase field simulations, Acta Materialia, 55 (2007) 2173-2182.

42. Ma, N., A. Kazaryan, S.A. Dregia, and Y. Wang, Computer simulation of texture evolution during grain growth: effect of boundary properties and initial microstructure, Acta Materialia, 52 (2004) 3869-3879.

43. Kazaryan, A., Y. Wang, S.A. Dregia, and B.R. Patton, Grain growth in anisotropic systems: comparison of effects of energy and mobility, Acta Materialia, 50 (2002) 24912502. 
44. Kazaryan, A., Y. Wang, S.A. Dregia, and B.R. Patton, Grain growth in systems with anisotropic boundary mobility: Analytical model and computer simulation, Physical Review B, 63 (2001) 184102.

45. Gruber, J., H.M. Miller, T.D. Hoffmann, G.S. Rohrer, and A.D. Rollett, Misorientation texture development during grain growth. Part I: Simulation and experiment, Acta Materialia, 57 (2009) 6102-6112.

46. Gruber, J., A.D. Rollett, and G.S. Rohrer, Misorientation texture development during grain growth. Part II: Theory, Acta Materialia, 58 (2010) 14-19.

47. Knapp, J.A. and D.M. Follstaedt, Hall-Petch relationship in pulsed-laser deposited nickel films, J. Mater. Res., 19 (2004) 218-227.

48. Keller, R.R. and R.H. Geiss, Transmission EBSD from $10 \mathrm{~nm}$ domains in a scanning electron microscope, Journal of Microscopy, 245 (2012) 245-251.

49. Trimby, P.W., Orientation mapping of nanostructured materials using transmission Kikuchi diffraction in the scanning electron microscope, Ultramicroscopy, 120 (2012) 16-24.

50. Trimby, P.W., Y. Cao, Z. Chen, S. Han, K.J. Hemker, J. Lian, X. Liao, P. Rottmann, S. Samudrala, J. Sun, J.T. Wang, J. Wheeler, and J.M. Cairney, Characterizing deformed ultrafine-grained and nanocrystalline materials using transmission Kikuchi diffraction in a scanning electron microscope, Acta Materialia, 62 (2014) 69-80.

51. Lupinacci, A., J. Kacher, A. Eilenberg, A.A. Shapiro, P. Hosemann, and A.M. Minor, Cryogenic in situ microcompression testing of Sn, Acta Materialia, 78 (2014) 56-64.

52. Mortazavi, N., M. Esmaily, and M. Halvarsson, The capability of Transmission Kikuchi Diffraction technique for characterizing nano-grained oxide scales formed on a FeCrAl stainless steel, Materials Letters, 147 (2015) 42-45.

53. Wen, Y.-N. and J.-M. Zhang, Surface energy calculation of the fcc metals by using the MAEAM, Solid State Communications, 144 (2007) 163-167.

54. Olmsted, D.L., S.M. Foiles, and E.A. Holm, Survey of computed grain boundary properties in face-centered cubic metals: I. Grain boundary energy, Acta Materialia, 57 (2009) 3694-3703.

55. Park, N.-J., D.P. Field, M.M. Nowell, and P.R. Besser, Effect of film thickness on the evolution of annealing texture in sputtered copper films, Journal of Electronic Materials, 34 (2005) 1500-1508.

56. White, J.R., Annealing behaviour of thin evaporated gold films, Thin Solid Films, 22 (1974) 23-35.

57. Floro, J.A. and C.V. Thompson, Numerical analysis of interface energy-driven coarsening in thin films and its connection to grain growth, Acta Metallurgica et Materialia, 41 (1993) 1137-1147.

58. Jamshidian, M., P. Thamburaja, and T. Rabczuk, Modeling the effect of surface energy on stressed grain growth in cubic polycrystalline bodies, Scripta Materialia, 113 (2016) 209-213.

59. Mahajan, S., C.S. Pande, M.A. Imam, and B.B. Rath, Formation of annealing twins in FCC crystals, Acta Materialia, 45 (1997) 2633-2638.

60. Lin, B., Y. Jin, C.M. Hefferan, S.F. Li, J. Lind, R.M. Suter, M. Bernacki, N. Bozzolo, A.D. Rollett, and G.S. Rohrer, Observation of annealing twin nucleation at triple lines in nickel during grain growth, Acta Materialia, 99 (2015) 63-68. 